\title{
奇异值分解中考虑频率因素的矩阵维数*
}

\author{
赵学智 邵啟鹏 叶邦彦 陈统坚 \\ (华南理工大学机械与汽车工程学院 广州 510640)
}

\begin{abstract}
摘要: Hankel 矩阵的维数对于奇异值分解的信号处理效果有非常重要的影响, 传统的维数没有考虑信号中的频率成分, 这是 不合理的。通过对频率因素的分析, 提出一种确定矩阵维数的最小公倍数法, 将原始信号中各频率成分的周期的最小公倍数 作为基数, Hankel 矩阵的行数和列数必须同时为这个基数的整数倍, 并在这一必要条件下使 Hankel 矩阵的维数最大, 由此 通过优化得到了最佳的矩阵行、列数。对模拟信号和转子振动信号的处理实例结果表明, 与传统的最大维数法相比, 在最小 公倍数法确定的矩阵维数下, 奇异值分解的计算量要小得多, 但是却可以获得波形误差更小的信号分解结果。
\end{abstract}

关键词: 奇异值分解; 矩阵维数; 频率周期; 最小公倍数; 信号分离

中图分类号: TN911

\section{Matrix Dimension Considering Frequency Factor in Singular Value Decomposition}

\author{
ZHAO Xuezhi SHAO Qipeng YE Bangyan CHEN Tongjian
}

(School of Mechanical and Automotive Engineering, South China University of Technology, Guangzhou 510640)

\begin{abstract}
The dimension of Hankel matrix has a very important influence on the signal processing effect of singular value decomposition (SVD). The traditional matrix dimension does not consider the frequency components in the signal and this is unreasonable. A least common multiple method is put forward to determine the matrix dimension based on the analysis for the frequency factor, and in this method, the least common multiple of the periods of the all frequency components in the original signal is used as a base number, and the row and column number of Hankel matrix must be the integer multiple of this base number, under this necessary condition, the dimension of Hankel matrix should be maximized, and then the optimal row number and column number are obtained by the optimization computation. The processing examples of simulation signal and rotor vibration signal are provided, which show that, under the matrix dimension determined by the least common multiple method, the calculation amount of SVD is much smaller, but waveform error of the decomposition results is much smaller than the ones of the traditional maximum dimension method.
\end{abstract}

Key words: singular value decomposition; matrix dimension; frequency period; least common multiple; signal separation

\section{0 前言}

奇异值分解(Singular value decomposition, SVD) 在信号处理和故障诊断领域有广泛的应用。SVD 的 一个突出优点是可以在噪声背景下提取到原始信号 中的特征信息, 如在材料无损探伤中, SVD 可以在 噪声干扰较严重的磁致伸缩导波信号中提取到其中 的材料损伤信号 ${ }^{[1]}$, 在机械故障诊断中, SVD 也常 常被用来提取故障特征, 如在噪声环境下对水轮机

* 国家自然科学基金(51375178、51875216)和广东省自然科学基金 (No.2018A030310017)资助项目。20190126 收到初稿, 20190702 收到修 改稿
故障调频特征的提取 ${ }^{[2]}$ 、对轴承振动故障特征的提 取 ${ }^{[3]}$ 、对电机振动故障特征的提取 ${ }^{[4]}$ 等, 此外, SVD 还被用于生物医学信号的特征提取, 如在噪声背景 下提取心电信号的 P-波、Q-波、T-波等特征信息 ${ }^{[5]}$ 。

SVD 在噪声背景下提取信号特征的能力实际 上来自于 SVD 的消噪能力, 对于一个含噪的信号, 利用此信号构造 Hankel 矩阵, 通过 SVD 处理后可 以将原信号分解为有用信号部分和噪声部分 ${ }^{[6-9]}$, 然 后选择合适的奇异值进行 SVD 重构, 就可以消除原 始信号中的噪声, 提取到有用的特征信息。在这个 过程中有两个问题最为关键: 一个是如何选择有效 奇异值, 而另一个就是如何确定矩阵的维数, 这两 个问题决定着 SVD 的消噪和特征提取效果。关于有 
效奇异值的选择, 已经有较多方法被提出来解决这 一问题, 如奇异熵法 ${ }^{[9]}$ 、奇异值差分谱法 ${ }^{[10]}$ 、曲率 谱法 ${ }^{[11]}$ 等, 本文不拟再探讨这一问题。而关于矩阵 的维数, 现在一般的做法是在确定的信号长度下, 使矩阵的维数达到最大 ${ }^{[1-12]}$, 这种方法可称之为最 大维数法。事实证明, 这种矩阵维数确实可以使 SVD 获得良好的消噪和特征提取效果, 但是本文研 究结果表明, 这并不是一种最佳的矩阵维数, 还存 在着一种更好的矩阵维数。在工程实际中, 各种不 同信号中的频率成分千差万别, 但最大维数法只考 虑了信号的长度, 却并没有考虑信号中的频率因素, 矩阵的维数只由原始信号的长度确定, 而与原始信 号中的频率成分没有任何关系, 这不能不说是一个 问题。作者通过研究, 发现了一种更好的矩阵维数 确定方法, 这种方法既考虑了信号的长度因素, 也 考虑了信号中的频率因素, 事实证明这是一种比传 统最大维数法更好的方法, 这就是基于频率周期最 小公倍数的矩阵维数确定法。文中论述了这一方法 的原理, 并通过模拟信号和转子振动信号的处理实 例证明了这种矩阵维数的效果比传统的最大维数效 果要好。这种最小公倍数法得到的矩阵维数比传统 的最大维数要小, 因此 SVD 的计算量要小, 但却可 获得比最大维数法波形失真更小的信号处理结果。

\section{1 确定矩阵维数的最小公倍数法}

SVD 本身是一种对矩阵的正交化分解方法, 将 SVD 应用于信号处理时, 首先必须利用待处理信号 构造一个矩阵, 这矩阵通常都是 Hankel 矩阵 ${ }^{[6-12]}$ 。 对于长度为 $N$ 的信号序列 $x(i), i=0,1,2, \cdots, N-1$, 可以构造 Hankel 矩阵 $\boldsymbol{H}$ 如下 ${ }^{[12]}$

$$
\boldsymbol{H}=\left(\begin{array}{cccc}
x(0) & x(1) & \cdots & x(n-1) \\
x(1) & x(2) & \cdots & x(n) \\
\vdots & \vdots & & \vdots \\
x(N-n) & x(N-n+1) & \cdots & x(N-1)
\end{array}\right)
$$

从式(1)可见，矩阵 $\boldsymbol{H}$ 的列数为 $n, n<N$, 而行数 $m=N-n+1$, 行数 $m$ 和列数 $n$ 存在以下制约关系

$$
m+n-1=N
$$

对式(1)所示的 Hankel 矩阵进行奇异值分解, 可以将
其分解为两个正交矩阵和一个对角矩阵的乘积, 如 式(3)所示

$$
\boldsymbol{H}=\boldsymbol{U} \boldsymbol{D} \boldsymbol{V}^{\mathrm{T}}
$$

式中, $\boldsymbol{U}$ 是 $m \times m$ 的正交矩阵, $\boldsymbol{V}$ 是 $n \times n$ 的正交矩 阵 ${ }^{[13]}$, 而 $\boldsymbol{D}$ 是一个 $m \times n$ 的对角矩阵，当 $m<n$ 时， $\boldsymbol{D}=\left(\operatorname{diag}\left(\sigma_{1}, \sigma_{2}, \cdots, \sigma_{q}\right), \boldsymbol{O}\right)$; 而当 $m>n$ 时， $\boldsymbol{D}=\left(\operatorname{diag}\left(\sigma_{1}, \sigma_{2}, \cdots, \sigma_{q}\right), \boldsymbol{O}\right)^{\mathrm{T}}$, 其中 $\boldsymbol{O}$ 表示零矩阵, $q=\min (m, n)$, 且: $\sigma_{1} \geqslant \sigma_{2} \geqslant \cdots \geqslant \sigma_{q} \geqslant 0$, 它们就是 矩阵 $\boldsymbol{H}$ 的奇异值, 也是原始信号 $x(i)$ 的奇异值。

对原始信号进行奇异值分解后，再选取合适的 奇异值进行 SVD 重构, 就可以消除掉原信号中的噪 声或者提取到原信号中的特征信息。在这一过程中, 除了奇异值的选取外, 影响 SVD 信号处理效果的另 一个重要因素就是 Hankel 矩阵的行数和列数, 对这 一问题目前一般的做法是对于长度为 $N$ 的信号, 使 其构造的 Hankel 矩阵的维数为最大 ${ }^{[1-13]}$ 。因构造的 Hankel 矩阵不一定是方阵, 称其行、列数中的小者 即 $q=\min (m, n)$ 为 Hankel 矩阵的维数。最大维数法 按如下方式确定矩阵行、列数: 如果信号的长度 $N$ 为偶数, 取列数 $n=N / 2$ 、行数 $m=N / 2+1$ 来构造 Hankel 矩阵; 如果 $N$ 为奇数, 取列数 $n=(N+1) / 2$ 、 行数 $m=(N+1) / 2$ 来构造 Hankel 矩阵 ${ }^{[10-11]}$ 。应该 说, 这种矩阵维数确实可以使 SVD 取得不错的消噪 效果和特征提取效果, 但是我们在研究中发现, 这 并不是最佳的矩阵维数, 还存在一种更好的矩阵维 数, 在这种矩阵维数下 SVD 可以获得比最大维数更 好的信号处理效果。

最大维数法的问题是它没有考虑信号中频率成 分的影响, 决定矩阵维数的只有一个因素, 那就是 信号的长度，不管信号具有怎样的频率成分，只要 根据信号的长度构造最大维数的 Hankel 矩阵即可。 但是，工程实际中信号的频率成分千差万别，如果 有两个完全不同的信号, 它们只是长度相同而频率 却完全不同, 但它们构造的矩阵维数却完全相同, 这实际上是不合理的。一种更合理的矩阵维数应该 与信号中的频率相联系。对于含一个频率成分的信 号 $x(t)=a \cdot \sin (\omega \cdot t+\phi)$, 以采样周期 $T_{s}$ 采集 $N$ 点数 据, 得到离散序列 $x(i)=a \cdot \sin \left(\omega T_{s} \cdot i+\phi\right), i=0,1, \cdots$, $N-1$, 其构造的 $m \times n$ Hankel 矩阵为

$$
\boldsymbol{H}=\left(\begin{array}{cccc}
a \cdot \sin \left(\omega T_{s} \cdot 0+\phi\right) & a \cdot \sin \left(\omega T_{s} \cdot 1+\phi\right) & \cdots & a \cdot \sin \left[\omega T_{s} \cdot(n-1)+\phi\right] \\
a \cdot \sin \left(\omega T_{s} \cdot 1+\phi\right) & a \cdot \sin \left(\omega T_{s} \cdot 2+\phi\right) & \cdots & a \cdot \sin \left(\omega T_{s} \cdot n+\phi\right) \\
\vdots & \vdots & & \vdots \\
a \cdot \sin \left[\omega T_{s} \cdot(m-1)+\phi\right] & a \cdot \sin \left(\omega T_{s} \cdot m+\phi\right) & \cdots & a \cdot \sin \left[\omega T_{s} \cdot(N-1)+\phi\right]
\end{array}\right)
$$

从式(4)可见，矩阵的每一行都是一个正弦信 号, 矩阵的每一列也都是一个正弦信号, 其频率均
为 $\omega$ 。因此可设想, 在构造矩阵时, 如果每一行和 每一列都能取到频率 $\omega$ 的整数倍周期, 这样在后续 
对数据做周期延拓时, 在延拓节点处不会存在任何 突变, 实现了对数据的自然无痕延伸, 这样必然可 以减少由延拓带来的误差, 其信号处理效果应该优 于那些非整数周期的矩阵结构。

然而，对于含有多个频率的信号，为了确保矩 阵行、列数是所有频率的整数倍周期, 我们提出了 一种最小公倍数法来确定矩阵维数, 并采用优化方 法来确定具体的整数倍数。不失一般性, 设待处理 信号 $x(t)$ 中含有 $I$ 个频率成分，表示如下

$$
x(t)=\sum_{i=1}^{I} a_{i} \cdot \sin \left(\omega_{i} t+\phi_{i}\right)
$$

式中, $a_{i}$ 是第 $i$ 个频率成分 $\omega_{i}$ 的幅值，而 $\phi_{i}$ 是相位。 对 $x(t)$ 以采样周期 $T_{s}$ 采集 $N$ 点数据, 得到离散序列 $x(j), j=0,1, \cdots, N-1$, 现在考虑信号中的频率成分来 确定矩阵维数, 步骤如下。

(1) 计算信号中各个频率的周期 $T_{i}$

$$
T_{i}=\frac{2 \pi}{\omega_{i}} \quad i=1,2, \cdots, I
$$

(2) 求取各个周期 $T_{i}(i=1,2, \cdots, I)$ 的最小公倍 数 $T_{g}$, 并计算此最小公倍数所对应的数据点数 $G$

$$
G=\operatorname{int}\left(\frac{T_{g}}{T_{s}}\right)
$$

式中，int 表示取整，按四舍五入。

(3) 将 Hankel 矩阵的行数和列数都取为 $G$ 的整 数倍, 即

$$
\left\{\begin{array}{l}
m=b \cdot G \\
n=d \cdot G
\end{array}\right.
$$

式中, $b, d$ 是整数。

(4) 确定两个整数 $b$ 和 $d$ 。由式(2)知行数 $m$ 和 列数 $n$ 是相互约束的,一个增大, 另一个必然减小, 在满足式(2)、(8)的情况下, 确定矩阵维数的原则 是使 $\min (m, n)$ 取到最大值, 这是一个优化问题。为 解决此问题, 首先将式(8)中

$$
m=b \cdot G
$$

代入式(2)，得到

$$
n=N+1-b \cdot G
$$

式(10)表明列数 $n$ 与变量 $b$ 是一种直线关系，其斜 率为 $-G$, 而行数 $m=b \cdot G$, 此直线通过坐标原点、 斜率为 $G$ 。随着变量 $b$ 的增大, 这两条直线一增一 降, 如图 1 所示, 因此它们的交点就是最优的 $b$ 值。 而交点利用式(11)计算

$$
b G=N+1-b \cdot G
$$

则得到

$$
b=\frac{N+1}{2 G}
$$

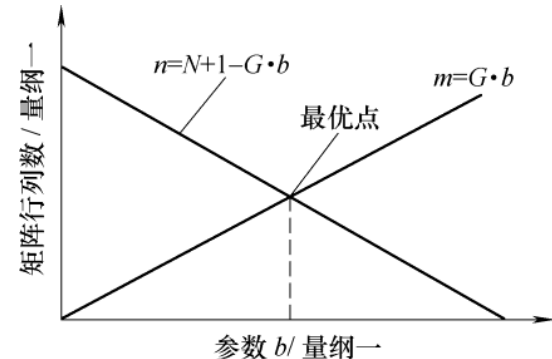

图 1 最优的 $b$ 值求取示意图

但是注意 $b$ 是一个整数，而 $(N+1) /(2 G)$ 不一定是 整数, 因此需对 $(N+1) /(2 G)$ 取整, 即

$$
b=\operatorname{int}\left(\frac{N+1}{2 G}\right)
$$

式中，int 表示取整，但是与式(7)中的取整不同，式 (13)中的取整是向下取整，即不四舍五入，因为如 果四舍五入, 最终处理的数据长度会超过原始长度 $N$ 。

再来求取另一个整数 $d$, 根据式(8)、(10)可得

$$
d \cdot G=N+1-b \cdot G
$$

因 $d$ 是整数，因此有

$$
d=\operatorname{int}\left(\frac{N+1-b \cdot G}{G}\right)
$$

式中, int 表示取整, 同样不四舍五入。最后将 $b$ 和 $d$ 代入式(8)就可得到矩阵行、列数 $m$ 和 $n$ 。

按照这样的方法确定的行、列数均是信号中各 个频率成分的周期的最小公倍数的整数倍。由于经 过取整操作, 所处理信号的长度将比原始长度 $N$ 略 小，变为

$$
L=m+n-1=(b+d) \cdot G-1
$$

$L$ 比信号的原始长度 $N$ 略小，但这种长度却可以使 矩阵的行、列数同时取到信号中各个频率成分的周 期的最小公倍数的整数倍, 我们称这种方法为最小 公倍数法。

最小公倍数法的物理意义是非常明显的，即矩 阵行数和列数都是信号中所有频率成分的整周期。 这种最小公倍数法确定的矩阵维数比传统的最大维 数法要小很多, 但是却可以获得比最大维数法波形 失真更小的信号处理结果。

\section{2 对模拟信号的处理实例}

设有信号 $x(t)=1.45 \cos (4 t+1.68)+1.12 \sin (6 t+0.76)+$ $0.92 \cos (16 t+1.65)+0.79 \sin (20 t+0.98)$, 对此信号以采 样周期 $T_{s}=0.02 \mathrm{~s}$ 采集 800 点数据, 并叠加白噪声, 信噪比 $3.54 \mathrm{~dB}$, 结果如图 2 所示。 


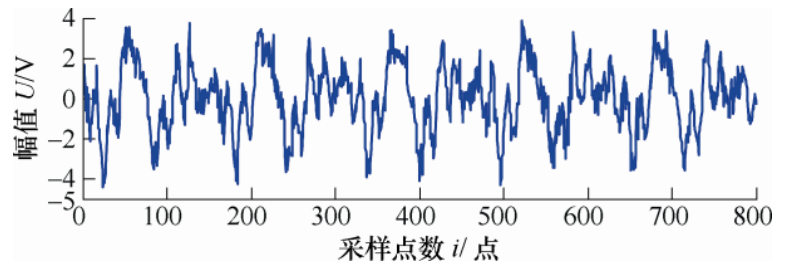

图 2 一个含噪的模拟信号

文献[14]的研究结果表明, 可以利用 SVD 来分 离出原信号中的各个频率成分。首先确定矩阵结构, 从原始信号可见, 其 4 个频率成分的周期依次为 $\pi / 2 、 \pi / 3 、 \pi / 8 、 \pi / 10$, 它们的最小公倍数 $T_{g}=\pi$, 其 对应的数据点数为

$$
G=T_{g} / T_{s}=\pi / 0.02=157.0796
$$

取整数部分得 $G=157$, 再根据式(13)计算得到行数 系数 $b=\operatorname{int}[(N+1) /(2 G)]=\operatorname{int}(801 / 314)=2$, 得到 $b$ 后, 再利用式(15)计算得到列数系数 $d=\operatorname{int}[(N+$ $1-b G) / G]=\operatorname{int}[(801-314) / 157]=3$ 则根据式 $(8)$, 可以确定 Hankel 矩阵的行数 $m=2 G=314$ 、列数 $n=3 G=471$, 这种情况下所处理的数据长度为 $L=m+n-1=784$, 比信号的原始长度 800 略小。

根据最小公倍数法, 利用原信号构造 $314 \times 471$ 的 Hankel 矩阵并进行奇异值分解。根据文献[14]的 结果, 一个频率成分对应两个非零奇异值, 选择这 两个非零奇异值进行 SVD 重构, 可以分离出相应的 频率成分。因原信号中有 4 个频率成分，它们产生 8 个非零奇异值, 而非零奇异值的大小与频率的幅

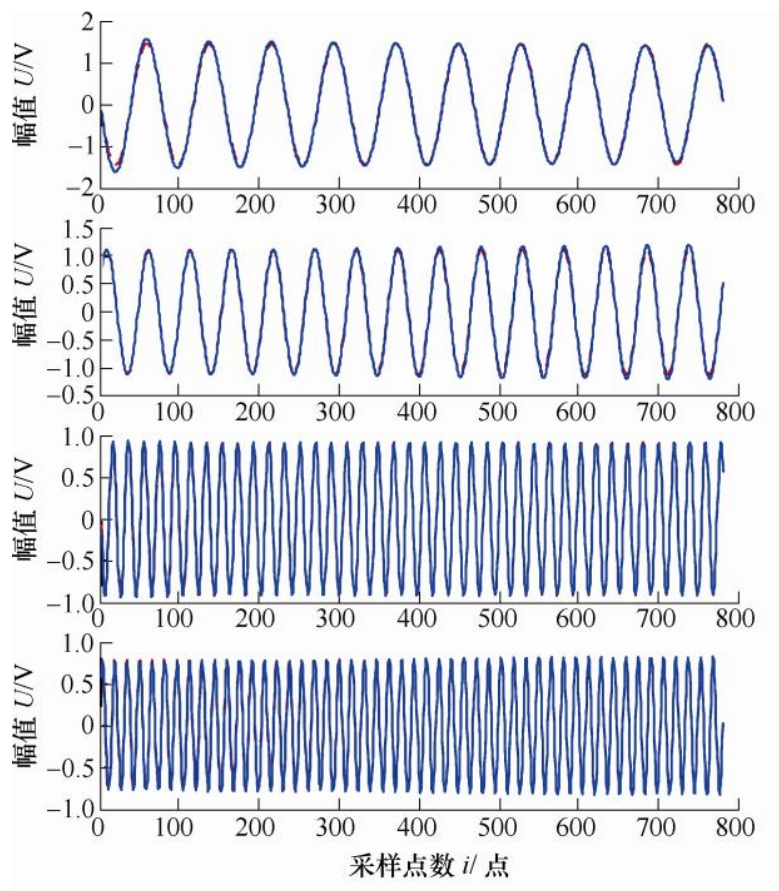

(a) 最小公倍数法 (矩阵大小: $314 \times 471$ )
值成正比，某一频率的幅值越大，其对应的两个非 零奇异值也就越可能排在前面 ${ }^{[14]}$, 因此原信号中的 4 个频率成分对应的就是前 8 个非零奇异值。现在 分别利用第 1 对、第 2 对、第 3 对和第 4 对奇异值 进行 SVD 重构，即有

$$
\boldsymbol{H}_{i}=\sum_{k=2 i-1}^{2 i} \sigma_{k} \boldsymbol{u}_{k} \boldsymbol{v}_{k}^{\mathrm{T}} \quad i=1,2,3,4
$$

采用平均法 ${ }^{[15]}$ 从矩阵 $\boldsymbol{H}_{i}(i=1,2,3,4)$ 中恢复出 信号, 结果如图 3a 所示, 可见原信号中的 4 个频 率分量被几乎完整地分离出来了, 分离出来的频率 分量依次是 $1.45 \cos (4 t+1.68) 、 1.12 \sin (6 t+0.76)$ 、 $0.92 \cos (16 t+1.65) 、 0.79 \sin (20 t+0.98)$ ，即幅值大 的频率分量先分离出来, 图中虚线是理想的频率 分量, 易见分离的结果和理想频率分量几乎完全 重合。

再来看传统的最大维数法的分离结果。原始信 号长度为 800, 根据最大维数法, 构造 $401 \times 400$ 的 Hankel 矩阵, 利用 SVD 对此矩阵进行同样的处理, 分离出的 4 个频率分量如图 $3 \mathrm{~b}$ 所示, 其中虚线是 理想的频率分量。从图 3 可见, 对于频率分量 $1.45 \cos (4 t+1.68)$ 和 $1.12 \sin (6 t+0.76)$, 即图中第一、 二个信号, 最大维数法的结果存在很大的波形失真, 而最小公倍数法的结果却和理想分量几乎完全重 合。对于图中后两个信号, 两种方法的分离效果看 起来差别不大。

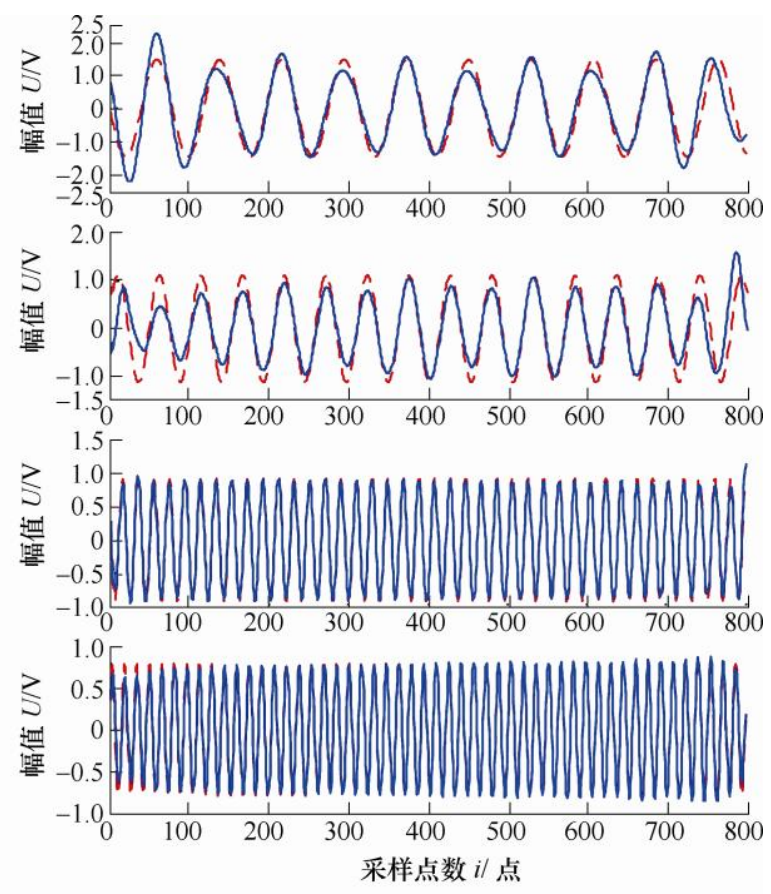

(b) 最大维数法 ( 矩阵大小: $401 \times 400$ )

图 3 最小公倍数法和最大维数法的处理结果对比

如果用 $y_{i}(j)$ 表示 SVD 分离出来的频率分量, $x_{i}(j)$ 表示原始信号中理想的频率分量, 则 SVD 分离结果
的波形误差为

$$
e_{i}(j)=x_{i}(j)-y_{i}(j) \quad i=1,2,3,4
$$


利用式(18)可以更直观地比较分离结果的误 差。根据式(18)计算两种维数下分离结果的波形误差, 结果如图 4 所示, 从上到下依次为 $1.45 \cos (4 t+1.68)$ 、 $1.12 \sin (6 t+0.76) 、 0.92 \cos (16 t+1.65) 、 0.79 \sin (20 t+$ 0.98) 的误差曲线, 其中虚线是最小公倍数法的 误差。进一步计算相对误差, 即 $\sum_{j}\left|e_{i}(j)\right| / \sum_{j}\left|x_{i}(j)\right|$, 结果如表 1 所示。可见对于后两个频率分量即 $0.92 \cos (16 t+1.65)$ 和 $0.79 \sin (20 t+0.98)$, 尽管两种方 法的结果看起来差不多, 但是最小公倍数法的误差 还是要小一些, 其他两个频率成分就更不用说了。

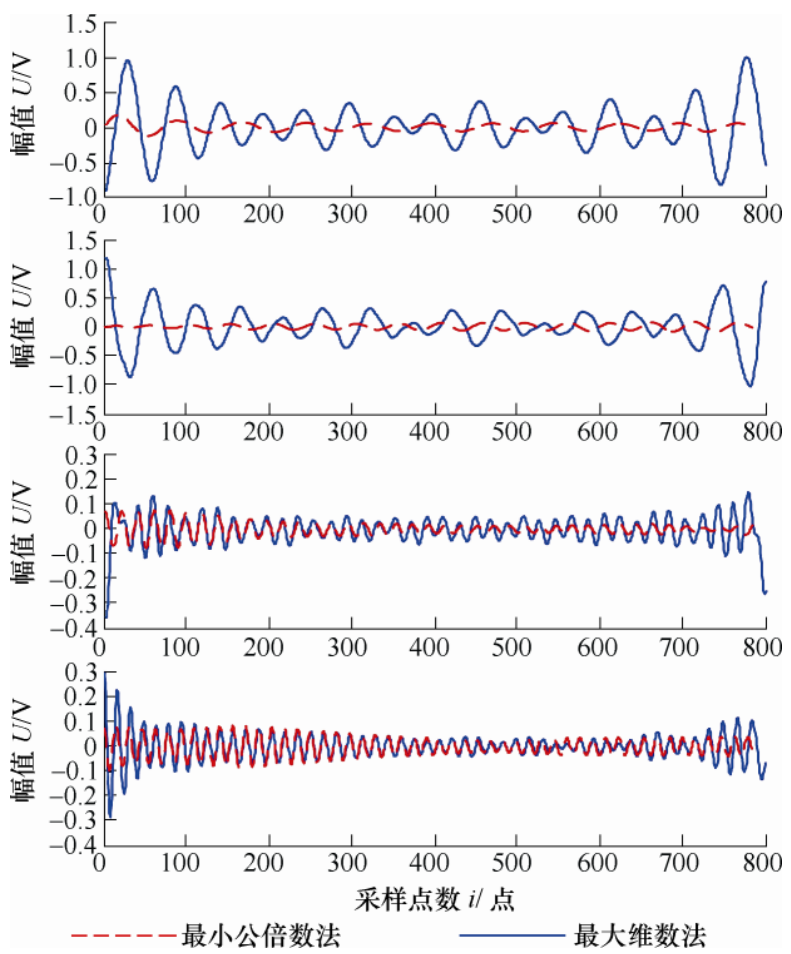

图 4 两种矩阵维数下分离出来的各频率的波形误差

\section{表 1 两种矩阵维数下各频率的波形失真相对误差}

\begin{tabular}{ccc}
\hline 频率分量 & 最小公倍数法 $(\%)$ & 最大维数法 $(\%)$ \\
\hline $1.45 \cos (4 t+1.68)$ & 4.80 & 27.43 \\
$1.12 \sin (6 t+0.76)$ & 5.14 & 32.58 \\
$0.92 \cos (16 t+1.65)$ & 3.27 & 6.92 \\
$0.79 \sin (20 t+0.98)$ & 6.28 & 7.74 \\
$1.24 \sin (3 t+0.25)$ & 6.70 & 25.63 \\
$1.15 \sin (7 t+0.78)$ & 6.18 & 25.47 \\
$1.02 \sin (13 t+1.16)$ & 3.85 & 6.71 \\
$0.82 \sin (19 t+1.85)$ & 9.94 & 10.45 \\
\hline
\end{tabular}

再来看另一个全是素数频率的信号。对于信号 $x(t)=1.24 \sin (3 t+0.25)+1.15 \sin (7 t+0.78)+1.02 \sin (13 t+$ $1.16)+0.82 \sin (19 t+1.85)$, 这个信号的特点是它的所 有频率全部都是素数。对于此信号以 $T_{s}=0.025 \mathrm{~s}$ 采 集 512 点数据, 并叠加白噪声, 信噪比 $3.15 \mathrm{~dB}$, 结 果如图 5 所示。

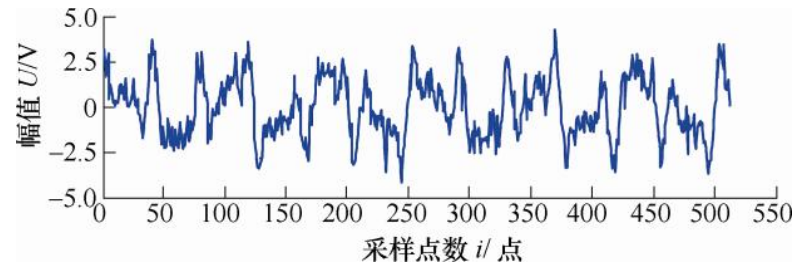

图 5 频率都是素数的含噪信号

利用最小公倍数法来分离各个素数频率, 各素 数频率的周期依次为 $2 \pi / 3 、 2 \pi / 7 、 2 \pi / 13 、 2 \pi / 19$, 显 然, 它们的最小公倍数 $T_{g}=2 \pi$, 实际上对于任何含 有素数频率的信号, $2 \pi$ 都是其各个频率的周期的最 小公倍数。此例中, $2 \pi$ 其对应的数据点数为 $G=T_{g} / T_{s}=2 \pi / 0.025=251.3$, 取整数部分得 $G=$ 251 , 则由式(13)可算得行数系数为

$$
b=\operatorname{int}[(N+1) /(2 G)]=\operatorname{int}(513 / 502)=1
$$

由式(15)算得列数系数为

$$
d=\operatorname{int}[(N+1-b G) / G]=\operatorname{int}[(513-251) / 251]=1
$$

因此, 利用最小公倍数法, 可确定矩阵行数为 $m=$ $1 G=251$, 而列数为 $n=1 G=251$, 此时所处理的数据 长度为 $L=m+n-1=501$ 。

根据最小公倍数法, 利用原信号构造 $251 \times 251$ 的 Hankel 矩阵, 并根据式(17)分离出各个频率, 结 果如图 6a 所示, 从上到下依次是 $1.24 \sin (3 t+0.25)$ 、 $1.15 \sin (7 t+0.78) 、 1.02 \sin (13 t+1.16) 、 0.82 \sin (19 t+$ 1.85), 其中虚线是理想的频率分量, 可见分离结果 和理想频率分量几乎完全重合。若采用最大维数法 来分离, 因原信号长度为 512 , 根据最大维数法, 构造的 Hankel 矩阵大小为 $257 \times 256$, 在此维数下的 分离结果如图 $6 \mathrm{~b}$ 所示, 可见对于第 $1 、 2$ 个信号, 即频率分量 $1.24 \sin (3 t+0.25)$ 和 $1.15 \sin (7 t+0.78)$, 最 大维数法产生了很大的波形失真, 而另两个频率 分量的波形失真小一些。根据公式 $\sum_{j}\left|e_{i}(j)\right| / \sum_{j} x_{i}(\not \supset \mid$

计算两种方法的波形失真相对误差, 结果也列在表 1 中(后 4 行), 可见对每个频率, 最小公倍数法都具 有更小的误差。

从图 3、图 6 以及表 1 的数据, 还可以发现一 个现象: 当频率较大时, 最大维数法分离出来的结 果波形失真较小, 基本和最小倍数法差不多, 如对 第一个模拟信号中的 $0.79 \sin (20 t+0.98)$, 最大维数法 的相对误差为 $7.74 \%$, 而最小公倍数法的相对误差 为 $6.28 \%$, 虽有差别, 但差别较小。而当频率较小 时, 最大维数法分离出来的结果波形失真较大, 最 小公倍数法的效果好很多, 如对第一个模拟信号中 的 $1.45 \cos (4 t+1.68)$, 最大维数法的相对误差为 $27.43 \%$, 而最小公倍数法的相对误差为 $4.80 \%$, 二 


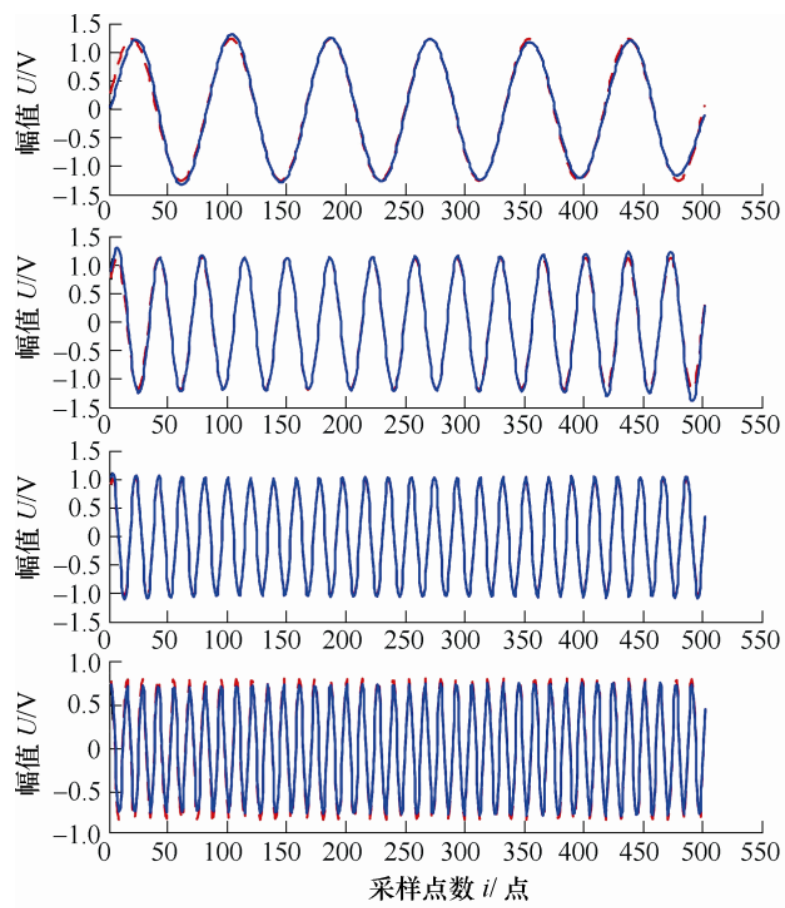

(a) 最小公倍数法 ( 矩阵大小: $251 \times 251$ )

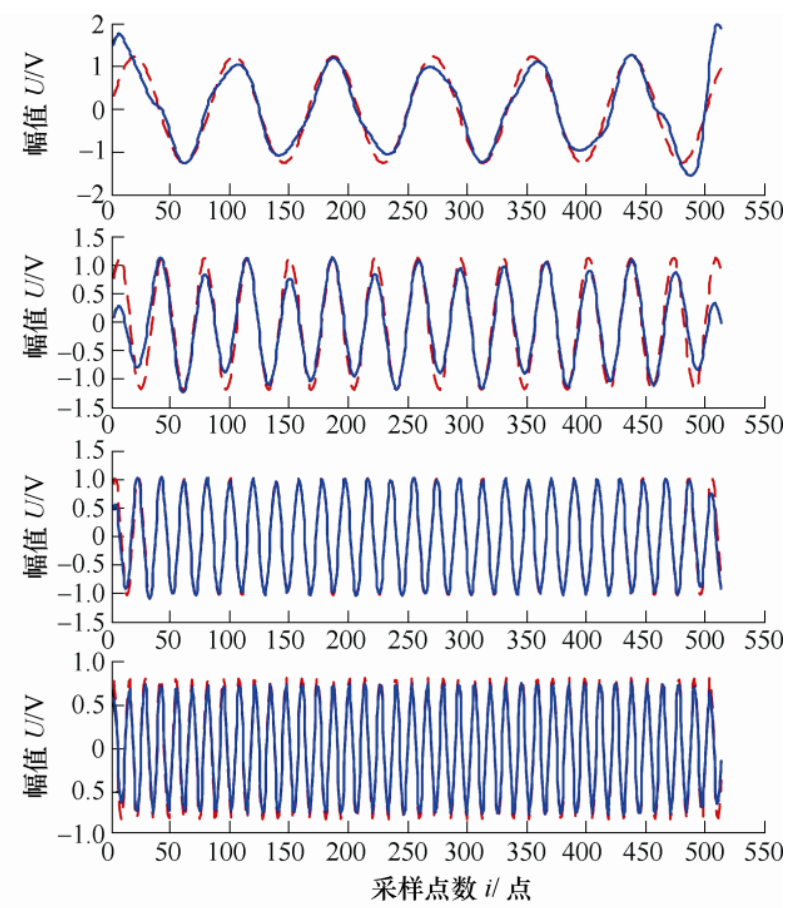

(b) 最大维数法 ( 矩阵大小: $257 \times 256$ )

图 6 对素数频率信号的分离结果对比

者的差别就很大了。第二个模拟信号的分离结果也 反映了这种现象。

发生这种现象的原因是, 当频率较大时, 此频率 的周期就较小, 而周期越小, 按最大维数法确定的矩 阵行、列数也就越容易近似为这个频率的整周期, 因 此分离出来的结果失真就小; 而当频率较小时, 此频 率的周期就较大, 对于这种较大的周期, 除非刻意按 照最小公倍数法来确定矩阵行、列, 否则矩阵的行、 列数就难以近似取到其整周期, 因而造成的波形失真 就大。在此二例中如果采用其他的频率, 也可得到类 似结果, 这里就不再给出更多的实例了。

然而, 而关于 SVD 对整个原始信号消噪后的波 形误差, 可以证明, 只要将 SVD 分离出来的各个频 率成分简单地叠加在一起, 就可得到原始信号的消 噪结果, 证明如下。

设原始信号 $x(t)=s(t)+\xi(t)$, 其中 $s(t)$ 是含有 $I$ 个频率成分的无噪声信号, 而 $\xi(t)$ 是噪声, 则 $x(t)$ 构造的矩阵 $\boldsymbol{H}$ 可表示为 $\boldsymbol{H}=\boldsymbol{H}_{s}+\boldsymbol{H}_{\xi}$, 其中 $\boldsymbol{H}_{\mathrm{s}}$ 是 $s(t)$ 构造而成, $\boldsymbol{H}_{\xi}$ 是 $\xi(t)$ 构造而成。对 $\boldsymbol{H}$ 进行 SVD, 并将 SVD 的方程式(1)中的两个正交矩阵 $\boldsymbol{U}$ 和 $\boldsymbol{V}$ 用 列矢量表示: $\boldsymbol{U}=\left(\boldsymbol{u}_{1}, \boldsymbol{u}_{2}, \cdots, \boldsymbol{u}_{m}\right), \boldsymbol{V}=\left(\boldsymbol{v}_{1}, \boldsymbol{v}_{2}, \cdots, \boldsymbol{v}_{n}\right)$, 则 $\boldsymbol{H}$ 的奇异值分解方程可以写成用列矢量 $\boldsymbol{u}_{i}$ 和 $\boldsymbol{v}_{i}$ 表示的形式

$$
\boldsymbol{H}=\boldsymbol{H}_{s}+\boldsymbol{H}_{\xi}=\sigma_{1} \boldsymbol{u}_{1} \boldsymbol{v}_{1}^{\mathrm{T}}+\sigma_{2} \boldsymbol{u}_{2} \boldsymbol{v}_{2}^{\mathrm{T}}+\cdots+\sigma_{q} \boldsymbol{u}_{q} \boldsymbol{v}_{q}^{\mathrm{T}}
$$

式中, $\boldsymbol{u}_{i}$ 是 $m \times 1$ 的列矢量, $\boldsymbol{v}_{i}$ 是 $n \times 1$ 的列矢量, $i=1,2, \cdots, q, q=\min (m, n)$ 。
根据文献[14]的结果，原始信号中的 $I$ 个频率一 共产生 $2 I$ 个非零奇异值, 这 $2 I$ 个奇异值排在奇异 值序列中的前面, 将这前 $2 I$ 个奇异值依次分成 $I$ 对 奇异值组合, 利用每对奇异值进行 SVD 重构, 得到

$$
\boldsymbol{H}_{i}=\sigma_{2 i-1} \boldsymbol{u}_{2 i-1} \boldsymbol{v}_{2 i-1}^{\mathrm{T}}+\sigma_{2 i} \boldsymbol{u}_{2 i} \boldsymbol{v}_{2 i}^{\mathrm{T}} \quad i=1,2, \cdots, I
$$

$\boldsymbol{H}_{i}$ 是一个 $m \times n$ 的矩阵, 从 $\boldsymbol{H}_{i}$ 中可以恢复出一个信 号 $y_{i}(t)$, 它就是原信号中的某一个频率成分, 这样 依次可将原信号中的 $I$ 个频率成分分离出来。而另 一方面，考虑到式(20)，则式(19)可表示为

$$
\begin{gathered}
\boldsymbol{H}_{s}+\boldsymbol{H}_{\xi}=\boldsymbol{H}_{1}+\boldsymbol{H}_{2}+\cdots+\boldsymbol{H}_{I}+ \\
\sigma_{2 I+1} \boldsymbol{u}_{2 I+1} \boldsymbol{v}_{2 I+1}^{\mathrm{T}}+\cdots+\sigma_{q} \boldsymbol{u}_{q} \boldsymbol{v}_{q}^{\mathrm{T}}
\end{gathered}
$$

在式(21)的右边, 从 $2 I+1$ 个奇异值开始的后续奇异 值都是噪声奇异值, 因此有

$$
\left\{\begin{array}{l}
\boldsymbol{H}_{s}=\boldsymbol{H}_{1}+\boldsymbol{H}_{2}+\cdots+\boldsymbol{H}_{I} \\
\boldsymbol{H}_{\xi}=\sigma_{2 I+1} \boldsymbol{u}_{2 I+1} \boldsymbol{v}_{2 I+1}^{\mathrm{T}}+\cdots+\sigma_{q} \boldsymbol{u}_{q} \boldsymbol{v}_{q}^{\mathrm{T}}
\end{array}\right.
$$

从式(22)中矩阵 $\boldsymbol{H}_{s}$ 的表达式可以得到

$$
s(t)=y_{1}(t)+y_{2}(t)+\cdots+y_{I}(t)
$$

式中, $y_{i}(t)$ 是从第 $i$ 个矩阵 $\boldsymbol{H}_{i}$ 中恢复出来的频率成 分, $i=1,2, \cdots, I$ 。可见, 将 SVD 分离出来的单个 频率简单叠加起来, 就可获得无噪声信号 $s(t)$ 。因 此, 在某一种矩阵维数下, 如果 SVD 分离出来的单 个频率成分的波形误差小，则这些频率成分叠加在 一起得到的信号的波形误差自然也小, 也即是原始 信号的消噪结果的波形误差也小。 
最后, 从计算量的角度来考虑, 对于一个 $m \times n$ 的矩阵, $q=\min (m, n)$, 直观上看, 共需要计算 $q$ 个 奇异值, 因此矩阵维数越小, SVD 需计算的奇异值 个数就越少, 而如果从 SVD 的计算过程深层次分 析 $^{[16-17]}$, SVD 的实现首先需要进行 $2(q-1)$ 次 Householder 变换将原始矩阵变为上双对角阵, 再利 用 $\mathrm{QR}$ 迭代将上双对角阵变为对角阵, 而对整个矩 阵完成一次 $\mathrm{QR}$ 迭代需要进行 $2(q-1)$ 次 Givens 变换。 而单次 Householder 变换和单次 Givens 变换的计算 量是与矩阵维数的立方成比例的, 当矩阵维数增大 时, 单次 Householder 变换和单次 Givens 变换的计 算量呈立方级速度增加 ${ }^{[16-17]}$ 。对于本节中第一个信 号, 最小公倍数法构造的矩阵为 $314 \times 471$, 则 $q=314$; 最大维数法构造的矩阵为 $401 \times 400$, 则 $q=400$, 比 最小公倍数法需多计算 172 次 Householder 变换和 172 次 Givens 变换, 而且在最大维数法下, 由于矩 阵维数大, 单次 Householder 变换和 Givens 变换的 计算量也远比最小公倍数法大得多, 因此最小公倍 数法的 SVD 计算量要少得多, 但却可以取得误差更 小的信号处理效果。

\section{3 转子振动信号分析}

在旋转机械中, 其频率成分一般都是转频以及 转频的高次谐波, 即 $\omega_{1} 、 2 \omega_{1} 、 3 \omega_{1} 、 \cdots$, 因为转频 的周期是其高次谐波周期的整数倍, 故各频率成分 的周期的最小公倍数就是转频的周期, 因此利用最 小公倍数法确定矩阵维数是很方便的。

在 INV1612 转子系统上用加速度计采集转子的 振动信号, 加速度计安装在轴承座上, 如图 7 所示。 试验时转速为 $1080 \mathrm{r} / \mathrm{min}$, 采样频率为 $400 \mathrm{~Hz}$, 采 样点数为 $N=512$, 得到的振动信号及其幅值谱如图 8 所示。从幅值谱来看, 此信号中在整个频带存在

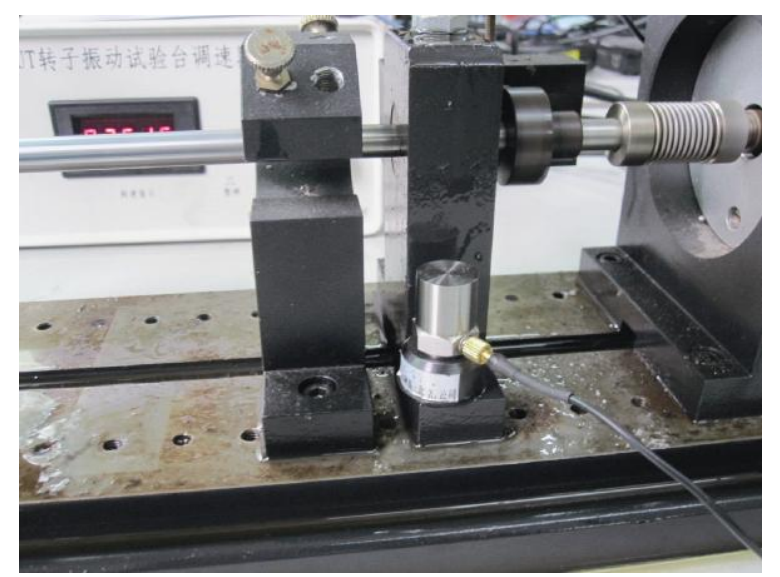

图 7 转子振动信息采集

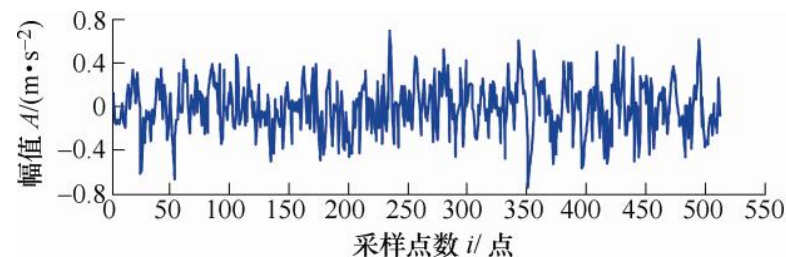

(a)

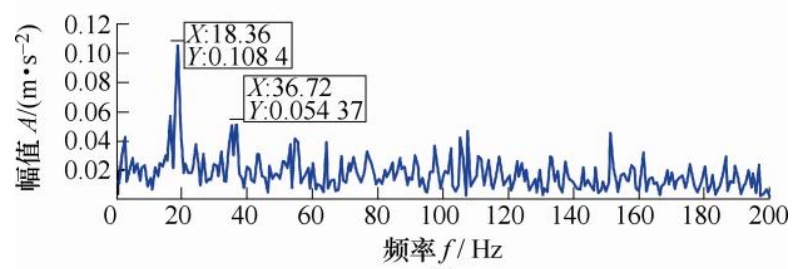

(b)

图 8 转子振动信号及其幅值谱

着较多的噪声, 但是两个主要频率是比较明显的。 一个是转频 $18.36 \mathrm{~Hz}$, 一个是二倍转频 $36.72 \mathrm{~Hz}$, 根据转子振动理论, 转频伴随着二倍转频这种频率 结构意味着转子对中不良。

频谱分析反映的是各频率的平均幅值, 但是实 际上有些频率成分的幅值可能是随时间变化的, 而 频谱分析结果无法反映频率幅值的变化。为了更准 确地分析设备的运转状态, 必须将时域和频域结合 起来分析 ${ }^{[18-19]}$, 而利用 SVD 可以提取各个频率成分 的时域波形，因此可以观察到各频率的幅值随时间 的变化过程, 从而更准确地对设备状态进行判断。

首先按最小公倍数法来确定矩阵维数, 因转频 的周期就是其高次谐波周期的最小公倍数, 根据式 (7)计算得到一个转频周期对应的数据点数为 $G=$ $\operatorname{int}\left(T_{g} / T_{s}\right)=\operatorname{int}(400 / 18.36)=22$, 再根据式(13)得到行 数的系数 $b=\operatorname{int}[(N+1) /(2 G)]=\operatorname{int}(513 / 44)=11$, 根 据式(15)得到列数的系数 $d=\operatorname{int}[(N+1-b G) / G]=$ $\operatorname{int}[(513-242) / 22]=12$, 因此, 可确定 Hankel 矩阵 的结构为行数 $m=b \cdot G=242$ 、列数 $n=d \cdot G=264$, 对 此矩阵进行 SVD 并分别取前 1 对、 2 对奇异值进行 重构, 得到的重构信号如图 9 所示。

从图 9 可见,第 1 对奇异值分离出来的是转频, 第 2 对奇异值分离出来的是二倍转频。从信号波形 来看, 这两个频率的波形并不是一成不变的, 转频 的幅值开始较小, 但随时间的增加而逐渐变大, 因 转频是不平衡引起的, 这表明转子旋转过程中不平 衡量在变大。而从二倍转频的波形来看, 这个频率 的幅值在后面却持续地减小, 这种减小几乎是线性 的, 到最后基本变为零了, 这说明二倍转频最后其 实基本消失了, 这种时域信号表明转子不对中程度 在旋转时是逐步改善的。对 INV1612 转子系统来说, 转子不对中来自于转子轴颈与两端滑动轴承的不对 中, 这种问题的产生主要与滑动轴承是否形成良好 
的油膜有直接关系。从二倍转频的幅值不断降低到 最后基本消失这一现象来看, 转子在旋转时滑动轴 承逐渐形成了良好的油膜, 因而导致了对中程度的

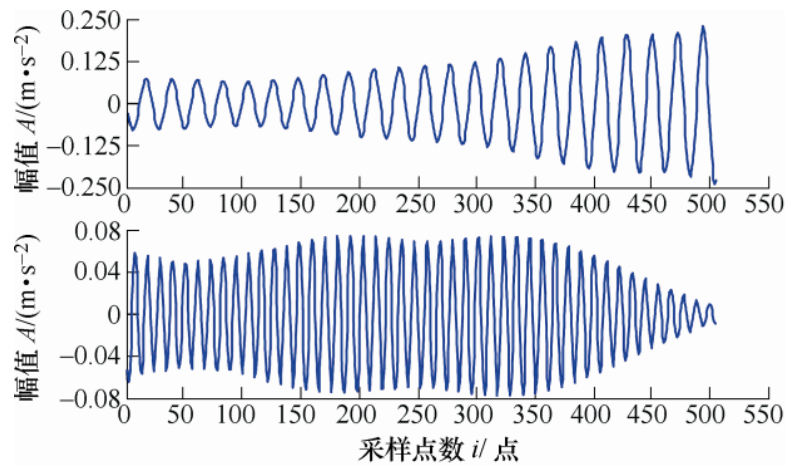

改善, 但是从原始振动信号及其幅值谱是看不出这 一现象的，而通过 SVD 对二倍频分量的分离，就可 以发现这一现象。

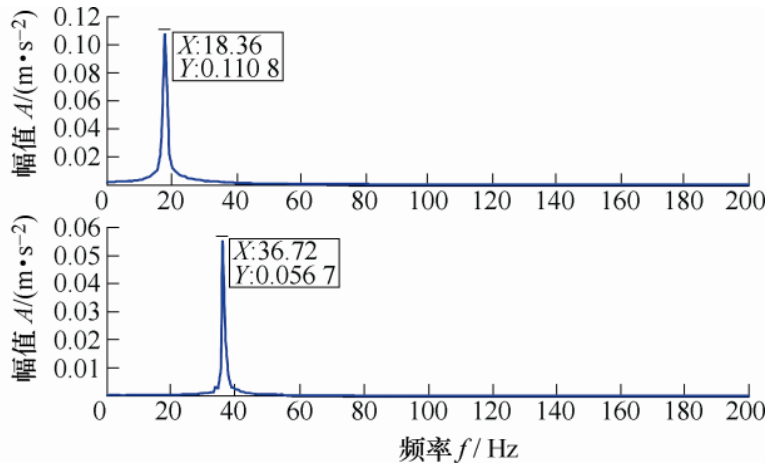

图 9 最小公倍数法确定的 $242 \times 264$ 矩阵下的信号分解结果

采用传统的最大维数法, 构造 $257 \times 256$ 的矩阵, 在此矩阵下得到的信号分离结果如图 10 所示。从信 号波形来看, 两种方法得到的波形看起来没有多大 区别, 因为不能像仿真信号那样有理想波形作为参 考, 因此这里无法对波形误差进行分析。但是, 从 模拟信号的结果来看, 我们有理由相信最小公倍数 法的结果更准确些, 而且必须注意到最小公倍数法

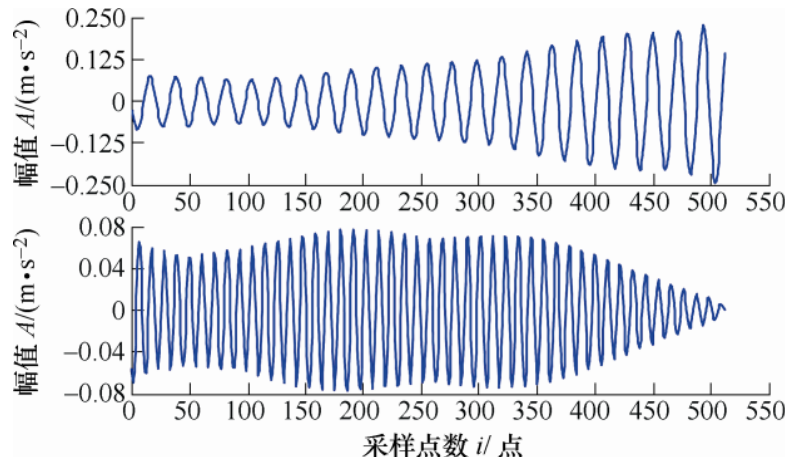

是在较小的矩阵维数下得到这种特征提取结果的, SVD 的计算量要少很多, 只需计算 242 个奇异值, 而最大维数法则需计算 256 个奇异值, 相应的, 其 SVD 计算需要多进行 28 次 Householder 变换和至少 多需要 28 次 Givens 变换, 因为通常 $\mathrm{QR}$ 迭代是需 要迭代多次的。用更小的计算量，取得更好的特征 提取效果，这样的方法是非常有意义的。

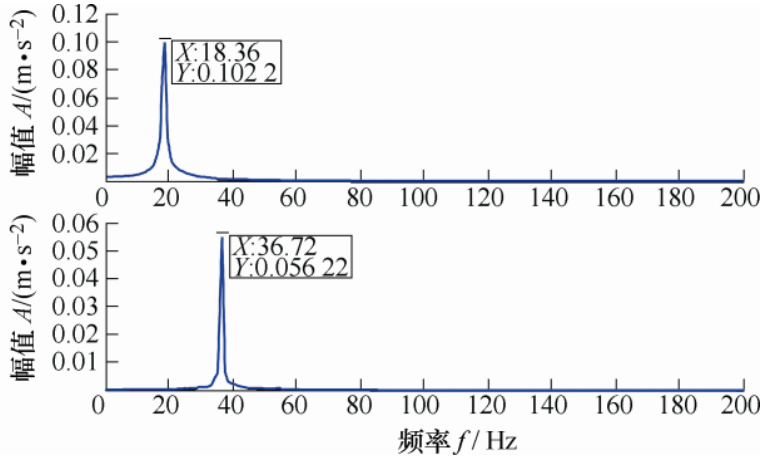

图 10 最大维数法确定的 $257 \times 256$ 矩阵下的信号分解结果

\section{4 结论}

(1) 矩阵维数是决定 SVD 信号处理效果的一个 重要因素, 对于确定的信号长度, 传统的做法是使 矩阵的维数达到最大, 但这并不是一种最好的矩阵 结构，它没有考虑信号中的频率信息，一种良好的 矩阵结构应该和原信号中的频率成分相联系。

(2) 提出了一种频率周期最小公倍数法来确定 矩阵结构, 将信号中各频率成分的周期的最小公倍 数作为基数, 在确保矩阵的行、列数是这一基数的 整数倍的情况下使矩阵维数达到最大, 并通过优化 方法得到了最佳的矩阵维数, 这种维数小于传统的 最大维数法确定的矩阵维数。
（3）最小公倍数法具有明显的物理意义，即矩 阵行数和列数必须同时为原始信号中各频率成分的 整周期。通过模拟信号和实际转子振动信号的处理 实例, 证明了在这种最小公倍数法确定的矩阵结构 下，SVD 提取的特征频率的波形误差小，其效果优 于传统的最大维数法, 但是计算量却比最大维数法 小得多。

\section{参 考 文 献}

[1] TANG Mingxi, WU Xinjun, CONG Ming, et al. A method based on SVD for detecting the defect using the magnetostrictive guided wave technique[J]. Mechanical Systems and Signal Processing, 2016, 70-71(3): 601-612.

[2] CHEN Shiqian, YANG Yang, WEI Kexiang, et al. 
Time-varying frequency-modulated component extraction based on parameterized demodulation and singular value decomposition[J]. IEEE Transactions on Instrumentation and Measurement, 2016, 65(2): 276-285.

[3] GOLAFSHAN R, SANLITURK K Y. SVD and Hankel matrix based de-noising approach for ball bearing fault detection and its assessment using artificial faults[J]. Mechanical Systems and Signal Processing, 2016, 70-71(3): 36-50.

[4] HERNANDEZ-VARGAS M , CABAL-YEPEZ E , GARCIA-PEREZ A. Real-time SVD-based detection of multiple combined faults in induction motors[J]. Computers \& Electrical Engineering, 2014, 40(7): 2193-2203.

[5] SHARMA L N. Information theoretic multiscale truncated SVD for multilead electrocardiogram[J]. Computer Methods and Programs in Biomedicine, 2016, 129(6): 109-116.

[6] ZHANG Xiaoming, TANG Jian, ZHANG Meijun, et al. Noise subspaces subtraction in SVD based on the difference of variance values[J]. Journal of Vibroengineering, 2016, 18(7): 4852-4861.

[7] HASSANPOUR H, ZEHTABIAN A, SADATI S J. Time domain signal enhancement based on an optimized singular vector denoising algorithm[J]. Digital Signal Processing, 2012, 22(5): 786-794.

[8] 王建国, 李健, 万旭东. 基于奇异值分解和局域均值分 解的滚动轴承故障特征提取方法 $[\mathrm{J}]$. 机械工程学报, 2015, 51(3): 104-109.

WANG Jianguo, LI Jian, WAN Xudong. Fault feature extraction method of rolling bearings based on singular value decomposition and local mean decomposition[J]. Journal of Mechanical Engineering, 2015, 51(3): 104-109.

[9] YANG Wenxian, PETER W T. Development of an advanced noise reduction method for vibration analysis based on singular value decomposition [J]. NDT\&E International, 2003, 36(6): 419-432.

[10] 赵学智, 叶邦彦, 陈统坚. 奇异值差分谱理论及其在车 床主轴箱故障诊断中的应用 $[\mathrm{J}]$. 机械工程学报, 2010, 46(1): 100-108.

ZHAO Xuezhi, YE Bangyan, CHEN Tongjian. Difference spectrum theory of singular value and its application to the fault diagnosis of headstock of lathe [J]. Journal of Mechanical Engineering, 2010，46(1): 100-108.

[11] 赵学智, 叶邦彦, 陈统坚. 基于奇异值曲率谱的有效奇 异值选择[J]. 华南理工大学学报, 2010, 38(6): 11-18. ZHAO Xuezhi, YE Bangyan, CHEN Tongjian. Selection of effective singular values based on curvature spectrum of singular values[J]. Journal of South China University of Technology, 2010, 38(6): 11-18.

[12] 丁建明, 林建辉, 赵洁. 高速列车万向轴动不平衡检测 的 EEMD-Hankel-SVD 方法[J]. 机械工程学报, 2015, 51(10): 143-151, 159.

DING Jianming, LIN Jianhui, ZHAO Jie. Detection of the dynamic imbalance with cardan shaft in high-speed train applying EEMD-Hankel-SVD[J]. Journal of Mechanical Engineering, 2015, 51(10): 143-151, 159.

[13] WANG Wei, LIAN Peiyuan, ZHANG Shuxin, et al. Effect of facet displacement on radiation field and its application for panel adjustment of large reflector antenna[J]. Chinese Journal of Mechanical Engineering, 2017, 30(2) : $578-586$.

[14] 赵学智, 聂振国, 叶邦彦, 等. 信号有效奇异值的数量 规律及其在特征提取中的应用 [J]. 振动工程学报, 2016, 29(3): 532-541.

ZHAO Xuezhi, NIE Zhenguo, YE Bangyan, et al. Number law of effective singular values of signal and its application to feature extraction[J]. Journal of Vibration Engineering, 2016, 29(3): 532-541.

[15] 赵学智, 叶邦彦. 分量形成方式对奇异值分解信号处理 效果的影响 $[\mathrm{J}]$. 上海交通大学学报, 2011，45(3): 368-374.

ZHAO Xuezhi, YE Bangyan. Influence of formation manner of component on signal processing effect of singular value decomposition[J]. Journal of Shanghai Jiaotong University, 2011，45(3): 368-374.

[16] 赵学智, 叶邦彦, 陈统坚. 大型矩阵奇异值分解的多次 分割双向收缩 QR 算法 $[\mathrm{J}]$. 华南理工大学学报, 2010, $38(1): 1-8$

ZHAO Xuezhi, YE Bangyan, CHEN Tongjian. Multipartition and double-directions shrink $\mathrm{QR}$ algorithm for singular value decomposition of large-scale matrix[J]. Journal of South China University of Technology, 2010, 38(1): $1-8$.

[17] 赵学智, 叶邦彦. 单向收缩 QR 算法在奇异值分解中的 收玫特性[J]. 电子科技大学学报, 2010, 39(5): 762-767. ZHAO Xuezhi, YE Bangyan. Convergence characteristic of single direction shrink $\mathrm{QR}$ algorithm in the singular value decomposition[J]. Journal of University of Electronic Science and Technology of China, 2010, 39(5): 762-767.

[18] 袁静, 訾艳阳, 倪修华, 等. 改进集成噪声重构经验模 式分解的微弱时频特征增强方法及应用 [J]. 机械工程 学报，2016，52(19): 88-94.

YUAN Jing, ZI Yanyang, NI Xiuhua, et al. Weak time-frequent feature enhancement method using 
improved ensemble noise-reconstructed empirical mode decomposition and its application[J]. Journal of Mechanical Engineering, 2016，52(19): 88-94.

[19] SHI Kunju, LIU Shulin, JIANG Chao, et al. Rolling bearing feature frequency extraction using extreme average envelope decomposition[J]. Chinese Journal of Mechanical Engineering, 2016, 29(5): 1029-1036.

作者简介: 赵学智, 男, 1970 年出生, 博士, 教授, 博士研究生导师。 主要研究方向为信号处理与机械故障诊断。

E-mail: mezhaoxz@scut.edu.cn 